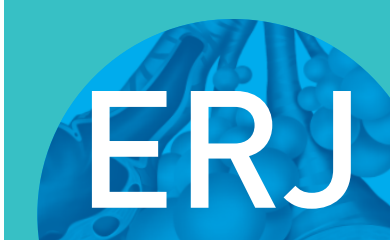

open research

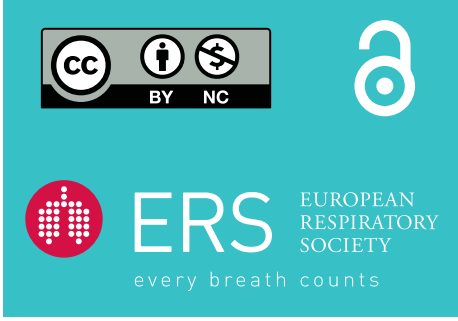

\section{Inhaled therapy reduces COPD mortality}

\author{
To the Editor:
}

The Global Initiative for Chronic Obstructive Lung Disease noted a lack of convincing evidence for a survival benefit with inhaled therapy in COPD despite relevant improvements in lung function, exacerbations and patient reported outcomes. Interestingly, recent studies investigating the role of triple therapy with long-acting $\beta_{2}$-agonist (LABA), long-acting muscarinic antagonist (LAMA) and inhaled glucocorticoid (ICS) showed that triple therapy could influence morbidity in patients with moderate to severe COPD.

The first large trial reporting mortality data in triple therapy was IMPACT (Informing the Pathway of COPD Treatment), published in 2018 [1]. The trial showed a hazard ratio for triple therapy versus the dual bronchiodilator umeclidinium-vilanterol of 0.58 (95\% CI 0.38-0.88). The hazard ratio for fluticasone furoate-vilanterol versus umeclidinium-vilanterol was 0.61 (95\% CI 0.40-0.93). Both effects were significant. Recently, a second large randomised controlled trial investigating triple therapy in COPD patients has been published [2]. This trial is named ETHOS (Efficacy and Safety of Triple Therapy in Obstructive Lung Disease), and showed a mortality benefit of triple therapy LABA, LAMA and ICS over dual therapy with LAMA-LABA [2]. The risk of death from any cause in the 320- $\mu$ g budesonide triple therapy group was $46 \%$ lower than that in the glycopyrrolate-formoterol group (hazard ratio $0.54,95 \%$ CI $0.34-0.87$ ). For the $160-\mu \mathrm{g}$ budesonide triple-therapy, the hazard ratio was 0.79 , missing statistical significance.

Although it should be remembered that mortality was not the primary endpoint in both trials, the data are of importance, since previous large controlled trials failed to show a significant effect on mortality with inhaled therapy in COPD even if compared to placebo. With $>16000$ patients included, the SUMMIT trial is the largest randomised controlled COPD trial ever. Patients with moderate airflow limitation $(\geqslant 50 \%$ and $\leqslant 70 \%$ predicted forced expiratory volume in $1 \mathrm{~s}$ ) and a history or an increased risk for cardiovascular disease were included. The primary endpoint, all-cause mortality, showed a $12 \%$ relative reduction with LABA-ICS compared to placebo, which was insignificant $(\mathrm{p}=0.137)$ [3]. Two preceding placebo-controlled studies $[4,5]$ with a statistically insignificant improvement in survival included patients with more severe COPD but less cardiovascular risk [6]. In the TORCH trial, $>6000$ COPD patients and a follow up of 3 years were included. The hazard ratio for the primary endpoint death with the LABA-ICS combination, as compared with placebo, was 0.825 , resulting in the legendary p-value of 0.052 [4]. The Uplift trial included nearly 6000 COPD patients with a follow up of 4 years [5]. The LAMA tiotropium bromide resulted in an insignificant decrease in the secondary endpoint mortality (hazard ratio 0.89, 95\% CI 0.79-1.02; $\mathrm{p}=0.09)$.

The failure of these and other older COPD trials with a placebo group might partly be attributed to the decrease in the rate of COPD exacerbation and COPD severity in controlled trials during the last two decades, making power calculations relying on historic data difficult [6]. Indeed, differences between study populations could also be important for the observed differences. Therefore, the significant mortality differences reported in the IMPACT and ETHOS trials discussed above are even more relevant. The question now arises, why does inhaled therapy reduce COPD mortality?

COPD exacerbations are followed by worsening lung function, reduced quality of life and increased mortality [7, 8]. Exacerbations increase the risk of myocardial infarction, stroke and death. Indeed, more patients with COPD die from cardiovascular disease than directly from respiratory failure itself [9].

@ERSpublications

Plotting exacerbation rate versus mortality rate for the treatment groups in two recent controlled trials investigating triple therapy in COPD, there is a significant positive correlation supporting a positive impact of inhaled therapy on mortality https://bit.ly/35jbEiN

Cite this article as: Andreas S, Taube C. Inhaled therapy reduces COPD mortality. ERJ Open Res 2020; 6: 00634-2020 [https://doi.org/10.1183/23120541.00634-2020]. 
FIGURE 1 Exacerbation and mortality in the ETHOS and IMPACT trials. $R^{2}=0.70, p=0.018$. B: budesonide; F: formeterol; FF: fluticasone furoate; G: glycopyrronium; U: umeclidinium; V: vilanterol.

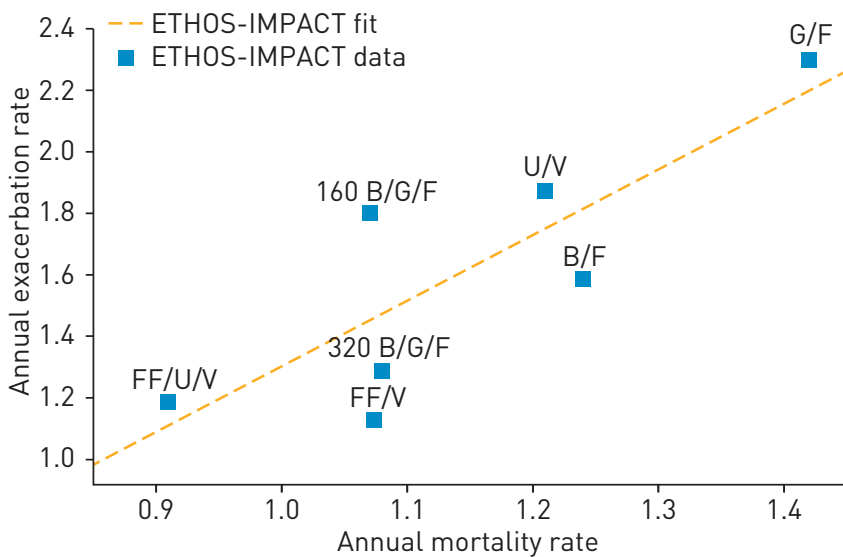

We thus reasoned that the reduction in COPD exacerbations in the IMPACT and ETHOS trial might determine the survival gain and plotted exacerbation rate versus mortality rate for the different treatment groups (figure 1). Indeed, this data shows a strong relationship between annual exacerbation rate and annual mortality rate (regression analysis: $\mathrm{R}^{2}=0.70, \mathrm{p}=0.018$ ). We acknowledge that we present grouped ecological data and not individual patient data, and therefore uncertainty remains on the strength of these effects. Nevertheless, these data, in combination with the well-known negative effects of exacerbations detailed above, are sufficient to claim that inhaled triple therapy reduces mortality in patients with at least moderate COPD and frequent exacerbations.

The authors of the ETHOS study suggested that the observed reduction in mortality with inhaled triple therapy was mediated mainly by the ICS [2]. Indeed, a beneficial effect of ICS on cardiovascular outcomes has been suggested previously [10]. However, it should be remembered that the Uplift trial, treating patients with either a LAMA or placebo over 4 years showed also some effect on the mortality as discussed above.

In conclusion, the reported mortality data of both triple-therapy studies fundamentally contribute to our understanding of the benefits of COPD therapy. It is time to concede that inhaled treatment not only has positive effects on lung physiology, symptoms and exacerbations, but also on prognosis in COPD patients.

Stefan Andreas $\oplus^{1,2,3}$ and Christian Taube ${ }^{4}$

${ }^{1}$ University Medical Center Göttingen, Göttingen, Germany. ${ }^{2}$ LungClinic Immenhausen, Immenhausen, Germany. ${ }^{3}$ Member of the German Center for Lung Research (DZL). ${ }^{4}$ Dept of Pulmonary Medicine, University Hospital Essen - Ruhrlandklinik, Essen, Germany.

Correspondence: Stefan Andreas, LungClinic Immenhausen, R. Koch Str. 3, Immenhausen 34376, Germany. E-mail: Stefan.andreas@med.uni-goettingen.de

Received: 1 Sept 2020 | Accepted: 4 Sept 2020

Conflict of interest: S. Andreas reports an Oskar Helene Prize during the conduct of the study; and grants and personal fees from Boehringer Ingelheim, grants from Pfizer, and personal fees from Novartis, AstraZeneca, GSK, Chiesi and Merini, outside the submitted work. C. Taube has nothing to disclose.

\section{References}

1 Lipson DA, Barnhart F, Brealey N, et al. Once-daily single-inhaler triple versus dual therapy in patients with COPD. N Engl J Med 2018; 378: 1671-1680.

2 Rabe KF, Martinez FJ, Ferguson GT, et al. Triple inhaled therapy at two glucocorticoid doses in moderate-to-very-severe COPD. N Engl J Med 2020; 383: 35-48.

3 Vestbo J, Anderson JA, Brook RD, et al. Fluticasone furoate and vilanterol and survival in chronic obstructive pulmonary disease with heightened cardiovascular risk (SUMMIT): a double-blind randomised controlled trial Lancet 2016; 387: 1817-1826.

4 Calverley PM, Anderson JA, Celli B, et al. Salmeterol and fluticasone propionate and survival in chronic obstructive pulmonary disease. N Engl J Med 2007; 356: 775-789.

5 Tashkin DP CB, Senn S, Burkhart D, et al. A 4-year trial of tiotropium in chronic obstructive pulmonary disease. N Engl J Med 2008; 359: 1543-1554.

6 Andreas S, Rover C, Heinz J, et al. Decline of COPD exacerbations in clinical trials over two decades a systematic review and meta-regression. Respir Res 2019; 20: 186. 
7 Suissa S, Dell'Aniello S, Ernst P. Long-term natural history of chronic obstructive pulmonary disease: severe exacerbations and mortality. Thorax 2012; 67: 957-963.

8 Celli BR, Wedzicha JA. Update on clinical aspects of chronic obstructive pulmonary disease. N Engl J Med 2019; 381: $1257-1266$.

9 Lahousse L, Niemeijer MN, van den Berg ME, et al. Chronic obstructive pulmonary disease and sudden cardiac death: the Rotterdam study. Eur Heart J 2015; 36: 1754-1761.

10 Lofdahl CG, Postma DS, Pride NB, et al. Possible protection by inhaled budesonide against ischaemic cardiac events in mild COPD. Eur Respir J 2007; 29: 1115-1119. 